\title{
CORRELAÇÕES CANÔNICAS ENTRE AS CARACTERÍSTICAS QUÍMICAS E ENERGÉTICAS DE RESÍDUOS LIGNOCELULÓSICOS
}

\author{
Thiago de Paula Protásioํㅡ, Gustavo Henrique Denzin Tonoli², Mário Guimarães Júnior³ ${ }^{3}$ Lina Bufalino ${ }^{4}$, \\ Allan Motta Couto ${ }^{4}$, Paulo Fernando Trugilho 5
}

(recebido: 17 de dezembro de 2010; aceito: 28 de fevereiro de 2012)

\begin{abstract}
RESUMO: A análise de correlação canônica é um procedimento estatístico multivariado que permite verificar as correlações lineares existentes entre dois grupos ou conjuntos de variáveis ( $\mathrm{X}$ e Y). No presente trabalho, objetivou-se realizar a análise de correlação canônica para verificar as associações existentes entre o grupo formado pelos teores de lignina, extrativos totais e o poder calorífico superior (PCS) de resíduos lignocelulósicos com o grupo formado pelos teores de seus componentes elementares (carbono, hidrogênio, nitrogênio e enxofre). Foram utilizados oito resíduos lignocelulósicos: maravalhas de eucalipto, pinus e cedro australiano; bagaço de cana; polpa celulósica residual de bambu; casca e pergaminho de café; resíduos da colheita do milho e casca de arroz. Apenas a primeira função canônica mostrou-se significativa, entretanto apresentou baixo $\mathrm{R}^{2}$ canônico. Encontrou-se uma tendência de altos teores de carbono, hidrogênio e enxofre e baixos teores de nitrogênio estarem associados com altos teores de extrativos totais dos resíduos lignocelulósicos avaliados. Esses resultados preliminares mostram que as correlações canônicas não foram eficientes para explicar as correlações existentes entre os componentes químicos elementares e os teores de lignina e o poder calorífico superior.
\end{abstract}

Palavras-chave: Estatística multivariada, função canônica, bioenergia.

\section{CANONICAL CORRELATIONS BETWEEN CHEMICAL AND ENERGETIC CHARACTERISTICS OF LIGNOCELLULOSIC WASTES}

\begin{abstract}
Canonical correlation analysis is a statistical multivariate procedure that allows analyzing linear correlation that may exist between two groups or sets of variables ( $X$ and $Y$ ). This paper aimed to provide canonical correlation analysis between a group comprised of lignin and total extractives contents and higher heating value (HHV) with a group of elemental components (carbon, hydrogen, nitrogen and sulfur) for lignocellulosic wastes. The following wastes were used: eucalyptus shavings; pine shavings; red cedar shavings; sugar cane bagasse; residual bamboo cellulose pulp; coffee husk and parchment; maize harvesting wastes; and rice husk. Only the first canonical function was significant, but it presented a low canonical $R^{2}$. High carbon, hydrogen and sulfur contents and low nitrogen contents seem to be related to high total extractives contents of the lignocellulosic wastes. The preliminary results found in this paper indicate that the canonical correlations were not efficient to explain the correlations between the chemical elemental components and lignin contents and higher heating values.
\end{abstract}

Key words: Multivariate statistics, canonical function, bioenergy.

\section{INTRODUÇÃO}

Na seleção de resíduos lignocelulósicos para a geração de bioenergia pode ser fundamental empregar técnicas estatísticas multivariadas para reconhecer as propriedades químicas e físicas mais importantes para um bom rendimento energético e para entender as correlações que podem ocorrer simultaneamente entre as variáveis de interesse.

A correlação canônica é uma técnica de análise de dados multivariada generalista, pois requer um menor número de restrições sobre os tipos de dados com os quais ela opera. De acordo com Hair Junior et al. (2005), há consenso entre pesquisadores de que a correlação

\footnotetext{
${ }^{1}$ Graduando em Engenharia Florestal - Universidade Federal de Lavras - Departamento de Ciências Florestais - Cx. P. 3037 - 37200-000 - Lavras, MG, Brasil - depaulaprotasio@gmail.com

${ }^{2}$ Engenheiro Florestal, Professor Doutor em Ciências e Engenharia de Materiais - Universidade Federal de Lavras - Departamento de Ciências Florestais - Cx. P. 3037 - 37200-000 - Lavras, MG, Brasil - gustavotonoli@dcf.ufla.br

${ }^{3}$ Licenciado Ciências e Matemática, Professor Mestre em Engenharia de Materiais - CEFET-MG - Campi Araxá - Departamento de Eletrônica Av. Ministro Olavo Drummond, 25, São Geraldo - 38180-510 - Araxá,MG, Brasil - mgjunior@araxa.cefetmg.br

${ }^{4}$ Engenheiros Florestais, Doutorandos em Ciência e Tecnologia da Madeira - Universidade Federal de Lavras - Departamento de Ciências Florestais Cx. P. 3037 -37200-000 - Lavras, MG, Brasil - linabufalino@gmail.com, allan_floresta@yahoo.com.br

${ }^{5}$ Engenheiro Florestal, Professor Doutor em Ciência Florestal - Universidade Federal de Lavras - Departamento de Ciências Florestais - Cx. P. 3037 37200-000 - Lavras, MG, Brasil - trugilho@dcf.ufla.br
}

Cerne, Lavras, v. 18, n. 3, p. 433-439, jul./set. 2012 
canônica é a técnica multivariada mais adequada nas situações em que se analisam muitas variáveis dependentes e independentes de um ou mais materiais.

Esse procedimento estatístico multivariado permite o exame da estrutura de relações lineares existentes entre dois grupos ou conjuntos de variáveis formados por variáveis métricas, de forma a maximizar a correlação entre eles. Para tanto, o procedimento envolve a obtenção de um conjunto de pesos para as variáveis dependentes e independentes (ABREU; VETTER, 1978; HAIR JUNIOR et al., 2009; MINGOTI, 2005).

De acordo com Hair Junior et al. (2005), a análise de correlação canônica consiste na obtenção de funções canônicas, sendo que cada função é composta por um par de variáveis estatísticas, representando as variáveis dependentes e independentes. O número máximo de funções canônicas que pode ser obtido é igual ao número de variáveis do menor conjunto de dados. Os autores ainda afirmam que o primeiro par de variáveis estatísticas canônicas é obtido de forma a ter-se a maior intercorrelação possível com os grupos de variáveis. O segundo par também é obtido para exibir a maior relação entre os grupos de variáveis analisadas, mas que não foi explicada pelo primeiro par e, assim, sucessivamente.

Nota-se então que as variáveis estatísticas canônicas (funções canônicas) são baseadas em variância residual e sua correlação decresce à medida que cada função canônica é calculada. Apesar de pouco explorada, Hair Junior et al. (2005) e Mingoti (2005) descrevem essa técnica como de alto potencial para fins preditivos.

Trugilho et al. (1997, 2003) demonstraram, respectivamente, o potencial da análise de correlações canônicas como ferramenta para a avaliação da qualidade da madeira de eucalipto para produção de carvão vegetal, e das características físicas, químicas e dimensionais de suas fibras. Entretanto, ainda existe uma ausência na literatura de estudos envolvendo a análise de correlação canônica entre características químicas / energéticas e a composição elementar de amostras de biomassa vegetal.

Nesse sentido, altos teores de carbono e hidrogênio são características importantes para a seleção de resíduos lignocelulósicos para a produção de bioenergia, em razão do valor energético desses elementos e da correlação positiva com o poder calorífico, enquanto que altos valores de oxigênio e nitrogênio decrescem o poder calorífico (HUANG et al., 2009; PAULA et al., 2011; PROTÁSIO et al., 2011). A alta proporção entre oxigênio e hidrogênio diminui o valor energético do material combustível (NORDIN, 1994). Além disso, é desejável que a biomassa apresente baixos teores de nitrogênio e enxofre, pois estes resultam em poluição ambiental (BILGEN; KAYGUSUZ, 2008; KUMAR et al., 2010).

Portanto, no presente trabalho, objetivou-se realizar a análise de correlação canônica para verificar as relações existentes entre o grupo de características químicas (lignina e extrativos totais) e energéticas (poder calorífico superior) de resíduos lignocelulósicos com o grupo formado pelos teores de seus respectivos componentes elementares $(\mathrm{C}, \mathrm{H}, \mathrm{N}$ e $\mathrm{S})$.

\section{MATERIAL E MÉTODOS}

\subsection{Características analisadas dos resíduos lignocelulósicos}

Foram utilizados os seguintes resíduos lignocelulósicos provenientes de biomassa florestal e agrícola: maravalhas de eucalipto (Eucalyptus urophylla de 6 anos de idade), maravalhas de cedro australiano (Toona ciliata de 18 anos de idade), maravalhas de pinus (Pinus sp. de 35 anos de idade), casca de arroz (Oryza sativa), bagaço de cana-de-açúcar (Saccharum officinarum), casca e pergaminho de café (Coffea arabica), resíduos da colheita do milho (Zea mays) (palha, sabugo, caule e folhas) e polpa celulósica residual de bambu (Bambusa vulgaris) oriunda da empresa Celulose e Papel de Pernambuco S/A (CEPASA).

Os resíduos lignocelulósicos foram moídos e a fração retida pela peneira de 270 mesh foi totalmente seca a $103 \pm 2^{\circ} \mathrm{C}$ para a quantificação dos teores $(\%$ em massa seca) de carbono, hidrogênio, nitrogênio e enxofre em um analisador universal da marca Elementar ${ }^{\circledR}$ (modelo Vario Micro Cube). $\mathrm{O}$ analisador utiliza hélio e oxigênio como gases de arraste e de ignição, respectivamente. $\mathrm{O}$ teor de oxigênio foi obtido por diferença, conforme recomendado por Bech et al. (2009), e apresentado na Equação 1:

$\mathrm{O}(\%)=100-\mathrm{C}(\%)-\mathrm{H}(\%)-\mathrm{N}(\%)-\mathrm{S}(\%)-\mathrm{CZ}(\%)$

em que: $\mathrm{O}$ é o teor de oxigênio (\%); C é o teor de carbono (\%); H é o teor de hidrogênio (\%); N é o teor de nitrogênio (\%); S é o teor de enxofre (\%) e CZ é o teor de cinzas (\%).

Os teores de extrativos totais e de lignina dos resíduos lignocelulósicos foram determinados seguindo os procedimentos das normas M3/69 (ASSOCIAÇÃO BRASILEIRA TÉCNICA DE CELULOSE E PAPEL ABTCP, 1974a) e M70/71 (ABTCP, 1974c). O teor de cinzas foi determinado segundo as diretrizes previstas na norma M11/77 (ABTCP, 1974b) com o uso de um forno tipo mufla.

Cerne, Lavras, v. 18, n. 3, p. 433-439, jul./set. 2012 
O poder calorífico superior (PCS) foi determinado em um calorímetro digital IKA C-200®, segundo as recomendações da norma NBR 08633 (ASSOCIAÇÃO BRASILEIRA DE NORMAS TÉCNICAS - ABNT, 1984).

Todas as análises foram realizadas considerandose 5 repetições, totalizando 40 observações por variável.

\subsection{Análise de correlação canônica}

Foram realizadas análises de correlação canônica para verificar as associações existentes entre um primeiro grupo de características químicas (lignina e extrativos totais) e energéticas (PCS) dos resíduos lignocelulósicos com um segundo grupo formado pelos teores dos seus componentes elementares (carbono, hidrogênio, nitrogênio e enxofre).

O primeiro grupo representa as variáveis dependentes (Y) e o segundo as independentes (X). Dessa forma, foi possível a determinação de 3 funções canônicas ou 3 pares de variáveis estatísticas canônicas. Foram estimadas as cargas canônicas, ou seja, as correlações entre as variáveis originais e suas respectivas variáveis estatísticas canônicas e as cargas canônicas cruzadas que representam a correlação entre uma variável original de um determinado grupo e a variável estatística canônica do outro grupo.

Para corrigir os possíveis efeitos dos resíduos lignocelulósicos, as correlações canônicas foram efetuadas considerando os resíduos (erros) provenientes de análises de variância realizadas para cada característica avaliada da biomassa vegetal. Além disso, antes da análise de correlação canônica, foi avaliada a existência de multicolinearidade entre as variáveis dos dois grupos considerados. $\mathrm{O}$ teor de oxigênio não foi incluído na análise de correlação canônica, pois é obtido pela diferença entre os componentes elementares.

Segundo Trugilho et al. (1997), a análise de correlação canônica se baseia na determinação de variáveis canônicas ortogonais em cada conjunto de variáveis e, por isso, as variáveis de cada conjunto devem ser linearmente independentes. Os autores ainda afirmam que se essa condição não for satisfeita será necessário descartar as variáveis redundantes ou que são combinações lineares das demais, justificando, assim, a não inclusão do teor de oxigênio na análise.

Utilizou-se o teste multivariado de significância Lambda de Wilks (aproximação da distribuição F) para avaliar a significância das raízes canônicas conjuntamente.
A quantidade de variância explicada, ou seja, o percentual de variância na variável estatística canônica dependente que pode ser explicada pela variável estatística canônica independente, e vice-versa, foi determinada elevando-se ao quadrado a correlação canônica $\left(\mathrm{R}^{2}\right.$ canônico).

Já a quantidade de variância compartilhada entre as variáveis observadas dependentes e independentes e as suas respectivas variáveis estatísticas canônicas foi determinada, elevando-se ao quadrado as cargas canônicas. O mesmo foi realizado para as cargas canônicas cruzadas com o intuito de estimar a variância compartilhada entre a variável dependente ou independente observada com a variável estatística canônica oposta.

O procedimento utilizado para as análises estatísticas foi baseado nas sugestões e recomendações de Hair Junior et al. $(2005,2009)$ e Trugilho et al. (1997, 2003).

Todas as análises foram realizadas utilizando-se o software estatístico R versão 2.11.0 (R DEVELOPMENT CORE TEAM, 2008), pacote CCA (GONZÁLEZ; DÉJEAN, 2009).

\section{RESULTADOS E DISCUSSÃO}

\subsection{Características analisadas dos resíduos lignocelulósicos}

$\mathrm{Na}$ Tabela 1, encontram-se os teores médios obtidos pela análise química elementar dos resíduos lignocelulósicos.

De forma geral, os resíduos provenientes da biomassa florestal apresentaram os maiores teores de carbono quando comparados aos resíduos agrícolas. Os teores de oxigênio da biomassa florestal estão próximos aos determinados para a biomassa agrícola. Esses valores encontrados são semelhantes àqueles obtidos por Kumar et al. (2010) para biomassa de eucalipto em diferentes idades. Observa-se, ainda, que o teor de hidrogênio apresentou pouca variação para a biomassa avaliada, sendo o maior valor e o menor valor encontrados para as maravalhas de pinus e casca de arroz, respectivamente.

Avaliando-se os teores de nitrogênio e enxofre, observa-se que os resíduos de origem florestal juntamente com a polpa celulósica residual de bambu apresentaram os menores percentuais desses elementos. Os coeficientes de variação foram baixos, com exceção daqueles determinados para o teor de enxofre da biomassa.

Cerne, Lavras, v. 18, n. 3, p. 433-439, jul./set. 2012 
Tabela 1 - Valores médios da análise elementar $(\mathrm{C}, \mathrm{H}, \mathrm{O}, \mathrm{N}$ e S) dos resíduos lignocelulósicos.

Table 1 - Average values of elemental analysis $(C, H, O, N$ and $S)$ for the lignocellulosic residues.

\begin{tabular}{lccccc}
\hline Resíduos Lignocelulósicos & $\mathrm{C}(\%)$ & $\mathrm{H}(\%)$ & $\mathrm{O}(\%)$ & $\mathrm{N}(\%)$ & $\mathrm{S}(\%)$ \\
\hline Maravalhas de eucalipto & $48,2^{(1,38)^{*}}$ & $6,36^{(1,27)}$ & $45,04^{(1,66)}$ & $0,07^{(12,30)}$ & $0,084^{(22,18)}$ \\
Maravalhas de pinus & $49,0^{(1,61)}$ & $6,65^{(1,82)}$ & $43,76^{(2,08)}$ & $0,09^{(14,17)}$ & $0,114^{(32,08)}$ \\
Maravalhas de cedro & $50,0^{(0,73)}$ & $6,26^{(1,01)}$ & $42,34^{(0,95)}$ & $0,27^{(4,16)}$ & $0,122^{(12,69)}$ \\
Bagaço de cana-de-açúcar & $46,8^{(2,35)}$ & $6,34^{(1,79)}$ & $45,44^{(2,51)}$ & $0,36^{(5,31)}$ & $0,134^{(31,01)}$ \\
Polpa celulósica de bambu & $45,2^{(0,34)}$ & $6,43^{(1,85)}$ & $46,67^{(0,64)}$ & $0,09^{(5,08)}$ & $0,048^{(17,43)}$ \\
Processamento de café & $46,5^{(0,82)}$ & $6,38^{(0,99)}$ & $39,86^{(1,09)}$ & $2,10^{(0,40)}$ & $0,236^{(12,90)}$ \\
Colheita do milho & $45,2^{(0,26)}$ & $6,43^{(2,13)}$ & $39,32^{(0,35)}$ & $1,99^{(1,31)}$ & $0,228^{(17,99)}$ \\
Casca de arroz & $36,0^{(0,44)}$ & $5,12^{(1,55)}$ & $41,56^{(0,67)}$ & $0,61^{(2,44)}$ & $0,194^{(31,55)}$ \\
\hline Média geral (\%) & 45,86 & 6,25 & 43,00 & 0,70 & 0,14 \\
\hline
\end{tabular}

C: carbono; H: hidrogênio; O: oxigênio; N: nitrogênio; S: enxofre; *Valores do coeficiente de variação amostral (\%).

A qualidade da biomassa para geração de bioenergia é conhecida pela quantidade de calor (energia) gerada por unidade de massa. O poder calorífico superior é um dos parâmetros importantes para diferenciar uma biomassa de outra quanto ao potencial para geração de energia e é dependente da composição química (celulose, hemiceluloses, lignina e extrativos) da biomassa avaliada (SHAFIZADEH, 1981). A lignina e os extrativos apresentam baixo grau de oxidação e alto calor de combustão em comparação com a celulose e as hemiceluloses (KUMAR et al., 1992), justificando seu uso no presente trabalho como variáveis dependentes.

$\mathrm{Na}$ Tabela 2, estão apresentados os valores médios para os teores de extrativos totais, lignina, cinzas e o poder calorífico superior (PCS) das biomassas avaliadas.

Observa-se que os resíduos da colheita do milho e bagaço de cana apresentaram os maiores valores de extrativos totais, sendo superiores a todos os resíduos avaliados. Já os resíduos do processamento de café apresentaram a maior quantidade de lignina. Quanto ao teor de cinzas, observa-se que a biomassa florestal apresentou, de maneira geral, valores inferiores aos determinados para a biomassa de origem agrícola.

Há relatos na literatura de que altos teores de extrativos totais (ARANTES, 2009) e altos teores de lignina (DEMIRBAS, 2001) podem influenciar positivamente no poder calorífico dos materiais. Já quanto maior o teor de cinzas menor o poder calorífico dos materiais lignocelulósicos, pois os minerais não participam do processo de combustão (BRAND, 2010).

Avaliando-se o poder calorífico superior dos resíduos lignocelulósicos observa-se que o menor valor encontrado foi para a casca de arroz, com valor muito similar ao encontrado por Huang et al. (2009). Isso ocorreu, possivelmente, em razão dos baixos teores de carbono e hidrogênio e altos teores de cinzas encontrados para esse material.

As maravalhas de pinus apresentaram o maior poder calorífico superior, apresentando valor similar ao encontrado por Sensöz (2003) para cascas de pinus. Certamente, os elevados valores de hidrogênio elementar e lignina encontrados para esse material contribuíram para a maximização do seu poder calorífico.

Observa-se, ainda, que todos os coeficientes de variação encontrados para a análise química (extrativos e lignina) e energética (PCS) foram baixos, com exceção do teor de extrativos totais da casca de arroz. Esse resultado pode ser explicado pela heterogeneidade do material analisado.

\subsection{Análise de correlação canônica}

Na Tabela 3, encontram-se as correlações canônicas, $o \mathrm{R}^{2}$ canônico e o teste multivariado de significância aplicado.

Apenas a função canônica 1 mostrou-se significativa a $1 \%$ de probabilidade e evidencia a dependência entre as características químicas e energéticas dos resíduos lignocelulósicos. Entretanto, o $\mathrm{R}^{2}$ canônico mostrouse consideravelmente baixo, ou seja, a quantidade de variância explicada $(43,01 \%)$ entre as variáveis estatísticas canônicas independente e dependente da função 1 não foi expressiva para os grupos de características analisadas.

Na Tabela 4, encontram-se as cargas canônicas e as cargas canônicas cruzadas determinadas para a função canônica 1, a única significativa dentre as estudadas.

Cerne, Lavras, v. 18, n. 3, p. 433-439, jul./set. 2012 
Tabela 2 - Valores médios de extrativos totais, lignina, cinzas e PCS dos resíduos lignocelulósicos.

Table 2 - Average values for total extractives, lignin, ashes and HHV for the lignocellulosic residues.

\begin{tabular}{|c|c|c|c|c|}
\hline Resíduos Lignocelulósicos & Extrativos totais $(\%)$ & Lignina (\%) & Cinzas $(\%)$ & PCS (kcal/kg) \\
\hline Maravalhas de eucalipto & $3,07^{(2,00)^{*}}$ & $25,65^{(0,80)}$ & $0,25^{(10,62)}$ & $4572,2^{(0,57)}$ \\
\hline Maravalhas de pinus & $2,61^{(11,98)}$ & $28,75^{(1,84)}$ & $0,39^{(11,70)}$ & $4864,2^{(1,18)}$ \\
\hline Bagaço de cana-de-açúcar & $16,59^{(1,55)}$ & $26,71^{(1,15)}$ & $0,93^{(4,78)}$ & $4511,8^{(0,61)}$ \\
\hline Maravalhas de cedro & $12,94^{(2,18)}$ & $25,90^{(2,21)}$ & $1,01^{(2,68)}$ & $4756,2^{(1,26)}$ \\
\hline Polpa celulósica de bambu & $3,00^{(8,56)}$ & $8,42^{(4,06)}$ & $1,56^{(8,70)}$ & $4192,4^{(0,91)}$ \\
\hline Processamento de café & $8,60^{(1,47)}$ & $31,03^{(2,08)}$ & $4,92^{(0,98)}$ & $4606,4^{(0,80)}$ \\
\hline Colheita do milho & $17,50^{(1,41)}$ & $22,85^{(0,54)}$ & $6,83^{(0,39)}$ & $4515,4^{(1,28)}$ \\
\hline Casca de arroz & $4,08^{(30,32)}$ & $28,94^{(2,32)}$ & $16,78^{(0,53)}$ & $3863,8^{(1,22)}$ \\
\hline Média geral (\%) & 8,55 & 24,78 & 4,08 & 4485,3 \\
\hline
\end{tabular}

PCS: poder calorífico superior ; *Valores do coeficiente de variação amostral (\%).

Tabela 3 - Teste multivariado Lambda de Wilks com aproximação da distribuição F.

Table 3-Multivariate test of Wilks'Lambda with approximation of $F$ distribution.

\begin{tabular}{cccccc}
\hline $\begin{array}{c}\text { Função } \\
\text { canônica }\end{array}$ & $\begin{array}{c}\text { Correlação } \\
\text { canônica }\end{array}$ & $\begin{array}{c}\mathrm{R}^{2} \\
\text { canônico }\end{array}$ & $\begin{array}{c}\text { Valor de } \\
\mathrm{F}^{\mathrm{a}}\end{array}$ & GL & p-valor \\
\hline 1 de 3 & 0,6558 & 0,4301 & $2,52^{*}$ & 88 & 0,0067 \\
2 de 3 & 0,4083 & 0,1667 & $1,34^{\text {ns }}$ & 68 & 0,2533 \\
3 de 3 & 0,1993 & 0,0397 & $0,72^{\text {ns }}$ & 35 & 0,4920 \\
\hline
\end{tabular}

Valor de $\mathrm{F}^{\mathrm{a}}$ : estatística $\mathrm{F}$ aproximada; GL: graus de liberdade do erro; *: significativo a $1 \%$ de significância; ns: não significativo a 5\% de significância.

Tabela 4 - Cargas canônicas e cargas canônicas cruzadas para a função canônica 1 .

Table 4 - Canonical loads and crossed canonical loads for the canonical function 1.

\begin{tabular}{lcc}
\hline Variáveis & Cargas canônicas & $\begin{array}{c}\text { Cargas canônicas } \\
\text { cruzadas }\end{array}$ \\
\cline { 1 - 3 } Dependentes (Y) & $-0,9411$ & $-0,6172$ \\
Extrativos totais & 0,2238 & 0,1467 \\
Lignina & 0,0687 & 0,0451 \\
Poder calorífico & & \\
superior & $-0,2241$ & $-0,1469$ \\
Independentes (X) & $-0,1374$ & $-0,0901$ \\
\hline Carbono & $-0,7551$ & $-0,4952$ \\
Hidrogênio & 0,1534 & 0,1006 \\
Enxofre & & \\
Nitrogênio & & \\
\hline
\end{tabular}

Segundo Hair Junior et al. (2005), as cargas canônicas (ou correlações estruturais canônicas) medem a correlação linear simples entre uma variável original observada no conjunto dependente ou independente e a variável estatística canônica do seu respectivo conjunto. Quanto maior a carga, mais importante é a variável para derivar a variável estatística canônica.

Observa-se que no grupo de variáveis dependentes (Y) apenas o teor de extrativos totais apresentou, em módulo, elevada carga canônica, o que resulta em alta variância compartilhada (aproximadamente 89\%). As demais variáveis apresentaram percentual de variável compartilhada estatisticamente desprezível. No grupo das variáveis independentes (X), observa-se expressiva carga canônica, em módulo, apenas para o teor de enxofre da biomassa residual analisada. Esse resultado gera um percentual de variância compartilhada entre essa variável e a variável estatística canônica do seu grupo de aproximadamente $57 \%$. As demais variáveis independentes observadas apresentaram percentual de variância compartilhada pouco expressiva.

Observa-se que os resultados encontrados para as cargas canônicas cruzadas são similares aos encontrados para as cargas canônicas. Nota-se que o teor de extrativos totais apresentou considerável correlação com a variável estatística canônica independente. Esse resultado confirma a expectativa de uma relação entre os componentes elementares e os extrativos totais. Entretanto, a quantidade de variância compartilhada entre essas duas variáveis é baixa e corresponde a aproximadamente $38 \%$. Analisando as cargas canônicas cruzadas estabelecidas para as variáveis independentes, observa-se que apenas o teor

Cerne, Lavras, v. 18, n. 3, p. 433-439, jul./set. 2012 
de enxofre apresentou um valor considerável, mas o percentual de variância compartilhada à semelhança do teor de extrativos totais, também foi baixo, cerca de $25 \%$.

Os resultados mostram que apenas 38\% da variância no teor de extrativos totais e $25 \%$ da variância no teor de enxofre são explicados pela função canônica 1. Analisando-se as cargas canônicas e as cargas canônicas cruzadas, percebe-se que há uma tendência de altos teores de carbono, hidrogênio e enxofre e baixos teores de nitrogênio estarem associados com altos teores de extrativos totais dos resíduos lignocelulósicos avaliados. As correlações encontradas entre os componentes elementares e o teor de lignina e o poder calorífico superior foram contrárias ao relatado na literatura (DEMIRBAS, 2001; HUANG et al., 2009; PAULA et al., 2011; PROTÁSIO et al., 2011; SHENG; AZEVEDO, 2005).

Além disso, tanto os extrativos totais quanto a lignina são compostos orgânicos, logo deveriam apresentar correlações diretas com os componentes elementares e vice-versa. Sheng e Azevedo (2005) correlacionaram o poder calorífico superior com os teores de carbono, hidrogênio e oxigênio em um único modelo estatístico linear múltiplo, evidenciando, assim, a associação linear entre essas variáveis.

Em estudos recentes, Paula et al. (2011) e Protásio et al. (2011) encontraram correlação positiva do poder calorífico com os teores de carbono e o hidrogênio, utilizando a correlação linear de Pearson. De forma semelhante, Huang et al. (2009) observaram correlação positiva do poder calorífico com o carbono e o hidrogênio, e correlação negativa com o nitrogênio. Paula et al. (2011) também obtiveram significativa associação linear entre os componentes elementares.

Lambert e Durand (1975 citados por HAIR JUNIOR et al., 2005) afirmam que as cargas canônicas podem estar sujeitas a considerável variabilidade de uma amostra para outra, o que sugere que as relações atribuídas a elas podem ser resultantes de fatores casuais extrínsecos, o que justificaria os resultados encontrados para as correlações entre os componentes elementares e o teor de lignina e o poder calorífico superior.

\section{CONCLUSÕES}

Analisando as cargas canônicas e as cargas canônicas cruzadas, percebe-se que há uma tendência de altos teores de carbono, hidrogênio e enxofre e baixos teores de nitrogênio estarem associados com altos teores de extrativos totais dos resíduos lignocelulósicos avaliados.
As correlações existentes entre os componentes químicos elementares e o teor de lignina e o poder calorífico superior não foram eficientemente explicadas pelas correlações canônicas, mas esses resultados preliminares indicam que esse tipo de análise multivariada pode ser utilizado para predição das variáveis dependentes e agrupamento dos resíduos lignocelulósicos em subgrupos homogêneos.

\section{AGRADECIMENTOS}

Os autores expressam seus sinceros agradecimentos aos Professores e Doutores Daniel Furtado Ferreira e Renato Ribeiro de Lima, da Universidade Federal de Lavras e ao CNPq, Fapemig e Capes pelo suporte financeiro concedido que foi fundamental para a execução desse trabalho.

\section{REFERÊNCIAS}

ABREU, M. A.; VETTER, D. A análise de relação entre conjuntos de variáveis na matriz geográfica: correlação canônica. In: FAISSOL, S. (Ed.). Tendências atuais na geografia urbana/regional: teorização e quantificação. Rio de Janeiro: IBGE, 1978. p. 133-144.

ARANTES, M. D. C. Variação nas características da madeira e do carvão de um clone de Eucalyptus grandis W. Hill ex Maiden x Eucalyptus urophylla S. T. Blake. 2009. 137 p. Tese (Doutorado em Ciência e Tecnologia da Madeira) - Universidade Federal de Lavras, Lavras, 2009.

ASSOCIAÇÃO BRASILEIRA DE NORMAS TÉCNICAS. NBR 8633: carvão vegetal: determinação do poder calorífico. Rio de Janeiro, 1984. 13 p.

ASSOCIAÇÃO BRASILEIRA TÉCNICA DE CELULOSE E PAPEL. M3/69: métodos de ensaio. São Paulo, 1974a. 8 p.

ASSOCIAÇÃO BRASILEIRA TÉCNICA DE CELULOSE E PAPEL. M11/77: teor de cinzas. São Paulo, 1974b. 8 p.

ASSOCIAÇÃO BRASILEIRA TÉCNICA DE CELULOSE E PAPEL. M70/71: métodos de ensaio. São Paulo, 1974c. 8 p.

BECH, N.; JENSEN, P. A.; DAM-JOHANSEN, K. Determining the elemental composition of fuels by bomb calorimetry and the inverse correlation of HHV with elemental composition. Biomass and Bioenergy, Oxford, v. 33, n. 3, p. 534-537, 2009.

Cerne, Lavras, v. 18, n. 3, p. 433-439, jul./set. 2012 
BILGEN, S.; KAYGUSUZ, K. The calculation of the chemical exergies of coal-based fuels by using the higher heating values. Applied Energy, London, v. 85, p. 776-785, 2008 .

BRAND, M. A. Energia de biomassa florestal. Rio de Janeiro: Interciência, 2010. 131 p.

DEMIRBAS, A. Relationships between lignin contents and heating values of biomass. Energy Conversion and Management, Oxford, v. 42, n. 2, p. 183-188, 2001.

GONZÁLEZ, I.; DÉJEAN, S. T. Canonical correlation analysis: R package version 2.11.1. Disponível em: $<\mathrm{http}: / / \mathrm{r}-$ forge.r-project.org>. Acesso em: 24 nov. 2010.

HAIR JUNIOR, J. F.; ANDERSON, R. E.; TATHAM, R. L.; BLACK, W. C. Análise multivariada de dados. Porto Alegre: Bookman, 2005. 593 p.

HAIR JUNIOR, J. F.; BLACK, W. C.; BABIN, B. J.; ANDERSON, R. E.; TATHAM, R. L. Análise multivariada de dados. Porto Alegre: Bookman, 2009. 688 p.

HUANG, C.; HAN, L.; YANG, Z.; LIU, X. Ultimate analysis and heating value prediction of straw by near infrared spectroscopy. Waste Management, Oxford, v. 29, p. 17931797, 2009.

KUMAR, M.; GUPTA, R. C.; SHARMA, T. Effect of carbonisation conditions on the yield and chemical composition of Acacia and Eucalyptus wood chars. Biomass and Bioenergy, Oxford, v. 3, p. 411-417, 1992.

KUMAR, R.; PANDEY, K. K.; CHANDRASHEKAR, N.; MOHAN, S. Effect of tree-age on calorific value and other fuel properties of Eucalyptus hybrid. Journal of Forestry Research, Oxford, v. 21, n. 4, p. 514-516, 2010.

MINGOTI, S. A. Análise de dados através de métodos de estatística multivariada: uma abordagem aplicada. Belo Horizonte: UFMG, 2005. 297 p.
NORDIN, A. Chemical and elemental characteristics of biomass fuels. Biomass and Bioenergy, Oxford, v. 6, p. 339-347, 1994.

PAULA, L. E. R.; TRUGILHO, P. F.; NAPOLI, A.; BIANCHI, M. L. Characterization of residues from plant biomass for use in energy generation. Cerne, Lavras, v. 17, n. 2, p. 237-246, abr./jun. 2011.

PROTÁSIO, T. P.; BUFALINO, L.; TONOLI, G. H. D.; COUTO, A. M.; TRUGILHO, P. F.; GUIMARÃES JÚNIOR, M. Relação entre o poder calorífico superior e os componentes elementares e minerais da biomassa vegetal. Pesquisa

Florestal Brasileira, Colombo, v. 31, n. 66, p. 122-133, 2011.

R DEVELOPMENT CORE TEAM. R: a language and environment for statistical computing. Vienna: R Foundation for Statistical Computing, 2008. Disponível em: $<$ http:// www.R-project.org >. Acesso em: $10 \mathrm{dez} .2010$.

SENSÖZ, S. Slow pyrolysis of wood barks from Pinus brutia Ten. and product compositions. Bioresource Technology, Essex, v. 89, p. 307-311, 2003.

SHAFIZADEH, F. Basic principles of direct combustion. In: SOFER, S. S.; ZABROSKY, O. R. (Ed.). Biomass conversion process for energy and fuels. New York: Plenum, 1981. p. 103-112.

SHENG, C.; AZEVEDO, J. L. T. Estimating the higher heating value of biomass fuels from basic analysis data. Biomass and Bioenergy, Oxford, v. 28, p. 499-507, 2005.

TRUGILHO, P. F.; LIMA, J. T.; MORI, F. A. Correlação canônica das características químicas e físicas da madeira de clones de Eucalyptus grandis e Eucalyptus urophylla. Cerne, Lavras, v. 9, n. 1, p. 66-80, 2003.

TRUGILHO, P. F.; VITAL, B. R.; REGAZZI, A. J.; GOMIDE, J. L. Aplicação da análise de correlação canônica na identificação de índices de qualidade da madeira de eucalipto para a produção de carvão vegetal. Revista Árvore, Viçosa, v. 21, n. 2, p. 259-267, mar./abr. 1997.

Cerne, Lavras, v. 18, n. 3, p. 433-439, jul./set. 2012 
